\title{
Transient Motion of an Elastic Cable Immersed in a Fluid
}

\author{
By H. Polachek, T. S. Walton, R. Mejia and C. Dawson
}

1. Introduction. In an earlier paper [1] the system of nonlinear differential equations governing the transient motion of a cable immersed in a fluid was formulated and solved by finite difference methods. Although the solution was framed in quite general terms and included a) motion in two dimensions, b) large displacements, c) forces due to weight of the cable, buoyancy, drag and virtual inertia, and d) nonuniform properties along the cable, the formulation was restricted in one sense. It was assumed that the cable was inextensible (cannot be stretched). In the present paper this restriction is removed. In addition to the conditions listed above, the cable is assumed to possess elastic properties.

2. Governing Equations. The equations governing the motion of an elastic cable moving in a fluid medium are equivalent to those derived in reference [1], with the exception of equation (2.6) $[1$, p. 32]. In the case of a cable with elastic properties this relation is replaced by an expression for Hooke's law. The complete set of governing differential equations is given here, with definitions of the notation used. A discrete representation of the cable (in which the distributed mass of the cable has been replaced by a series of masses $m_{j}$, attached to weightless elastic links) has again been used as in the original formulation of the problem. This is considered to be the simplest approach, since the method of finite differences, both in space and time dimensions, is used in the solution. Before listing the equations we also restate the generalized conditions which are used as the basis for the formulation of the problem:

a. The motion is transient (time-dependent) and takes place in two space dimensions.

b. The occurrence of large displacements from the equilibrium configuration of the cable is permitted.

c. The cable is elastic.

d. The cable is immersed in a fluid, requiring the inclusion of forces due to buoyancy, virtual inertia and drag.

e. The extremities of the cable may be at different levels with the cable sagging between the positions of support. Weight of the cable is taken into account.

f. Finally, provision is made for nonuniformity of the cable and for the suspension of concentrated loads, such as an anchor, at one or more positions along the cable.

The equations of motion may be written as follows:

$$
\begin{aligned}
I_{j} \ddot{x}_{j}-K_{j} \ddot{y}_{j} & =T_{j+1 / 2} \cos \theta_{j+1 / 2}-T_{j-1 / 2} \cos \theta_{j-1 / 2}+X_{j} \\
-K_{j} \ddot{x}_{j}+J_{j} \ddot{y}_{j} & =T_{j+1 / 2} \sin \theta_{j+1 / 2}-T_{j-1 / 2} \sin \theta_{j-1 / 2}+Y_{j}
\end{aligned}
$$

Received September 7, 1962. 
where

$$
\begin{aligned}
I_{j} & =m_{j}+\frac{1}{2}\left(e_{j+1 / 2} \sin ^{2} \theta_{j+1 / 2}+e_{j-1 / 2} \sin ^{2} \theta_{j-1 / 2}\right)+m_{j}{ }^{x} \\
J_{j} & =m_{j}+\frac{1}{2}\left(e_{j+1 / 2} \cos ^{2} \theta_{j+1 / 2}+e_{j-1 / 2} \cos ^{2} \theta_{j-1 / 2}\right)+m_{j}{ }^{Y} \\
K_{j} & =\frac{1}{2}\left(e_{j+1 / 2} \sin \theta_{j+1 / 2} \cos \theta_{j+1 / 2}+e_{j-1 / 2} \sin \theta_{j-1 / 2} \cos \theta_{j-1 / 2}\right) \\
X_{j} & =-\frac{1}{2}\left[D_{j+1 / 2} \sin \theta_{j+1 / 2}+D_{j-1 / 2} \sin \theta_{j-1 / 2}\right]+X_{j}^{*} \\
Y_{j} & =\frac{1}{2}\left[D_{j+1 / 2} \cos \theta_{j+1 / 2}+D_{j-1 / 2} \cos \theta_{j-1 / 2}\right]+Y_{j}^{*}-W_{j}-W_{j}^{*}
\end{aligned}
$$

and

$$
\begin{aligned}
& m_{j} \quad=\frac{1}{2}\left[\mu_{j+1 / 2} l_{j+1 / 2}+\mu_{j-1 / 2} l_{j-1 / 2}\right] \\
& e_{j+1 / 2}=\rho k_{j+1 / 2} l_{j+1 / 2} \sigma_{j+1 / 2} \\
& m_{j}^{X}=m_{j}^{*}+\rho V_{j}^{X} \\
& m_{j}{ }^{Y}=m_{j}^{*}+\rho V_{j}^{Y} \\
& \cos \theta_{j+1 / 2}=\left(x_{j+1}-x_{j}\right) /\left[\left(x_{j+1}-x_{j}\right)^{2}+\left(y_{j+1}-y_{j}\right)^{2}\right]^{1 / 2} \\
& \sin \theta_{j+1 / 2}=\left(y_{j+1}-y_{j}\right) /\left[\left(x_{j+1}-x_{j}\right)^{2}+\left(y_{j+1}-y_{j}\right)^{2}\right]^{1 / 2} \\
& W_{j} \quad=m_{j} g-\frac{1}{2} \rho g\left(l_{j+1 / 2} \sigma_{j+1 / 2}+l_{j-1 / 2} \sigma_{j-1 / 2}\right) \\
& W_{j}^{*} \quad=m_{j}^{*} g-\rho g V_{j}^{*} \\
& D_{j+1 / 2}=-f_{j+1 / 2}^{D} q_{j+1 / 2}\left|q_{j+1 / 2}\right| \\
& X_{j}^{*} \quad=-f_{j}^{{ }^{*}} u_{j}\left(\dot{x}_{j}-c\right) \\
& Y_{j}^{*} \quad=-f_{j}{ }^{{ }^{*}} u_{j} \dot{y}_{j} \\
& f_{j+1 / 2}^{D}=\frac{1}{2} \rho C_{j+1 / 2}^{D} l_{j+1 / 2} d_{j+1 / 2} \\
& q_{j+1 / 2}=-\frac{1}{2}\left[\left(\dot{x}_{j+1}-c\right)+\left(\dot{x}_{j}-c\right)\right] \sin \theta_{j+1 / 2}+\frac{1}{2}\left[\dot{y}_{j+1}+\dot{y}_{j}\right] \cos \theta_{j+1 / 2} \\
& f_{j}^{X} \quad=\frac{1}{2} \rho C_{j}^{X} S_{j}^{X} \\
& f_{j}{ }^{Y} \quad=\frac{1}{2} \rho C_{j}{ }^{Y} S_{j}{ }^{Y} \\
& u_{j} \quad=\left[\left(\dot{x}_{j}-c\right)^{2}+\dot{y}_{j}^{2}\right]^{1 / 2} \text {; } \\
& \text { (2) } T_{j+1 / 2}=\sigma_{j+1 / 2} E_{j+1 / 2}\left[\frac{\sqrt{\left(x_{j+1}-x_{j}\right)^{2}+\left(y_{j+1}-y_{j}\right)^{2}}}{l_{j+1 / 2}}-1\right] \text {. }
\end{aligned}
$$

In addition, in order to define a solution, boundary conditions

$$
\begin{array}{ll}
x_{0}=x_{0}(t), & y_{0}=y_{0}(t) \\
x_{s}=x_{s}(t), & y_{s}=y_{s}(t),
\end{array}
$$

and initial conditions,

$$
\begin{array}{lll}
x_{j}(0)=x_{j}{ }^{0}, & y_{j}(0)=y_{j}{ }^{0} & (j=1,2, \cdots s-1) \\
\dot{x}_{j}(0)=\dot{x}_{j}{ }^{0}, & \dot{y}_{j}(0)=\dot{y}_{j}{ }^{0} & (j=1,2, \cdots s-1)
\end{array}
$$

must be included, where the superscript index " 0 " is used to designate a value at the origin in time.

The definition of the symbols used are given below:

$C_{j+1 / 2}^{D} \quad$ Drag coefficient for segment of cable between stations $j$ and $j+1$

$C_{j}{ }_{j}^{X} \quad$ Resistance coefficient for horizontal motion of suspended prism

$C_{j}{ }^{\boldsymbol{Y}} \quad$ Resistance coefficient for vertical motion of suspended prism

$c \quad$ Velocity of uniform horizontal current

$D \quad$ Drag 
$d_{j+1 / 2}$ Diameter of segment of cable between stations $j$ and $j+1$

$E_{j+1 / 2} \quad$ Young's modulus of elasticity

$e_{j+1 / 2} \quad$ Virtual mass of entrained fluid between stations $j$ and $j+1$

$f_{j+1 / 2}^{D} \quad$ Drag factor for cable $=(\rho / 2) C_{j+1 / 2}^{D} l_{j+1 / 2} d_{j+1 / 2}$

$f_{j}{ }^{X} \quad$ Horizontal drag factor for suspended prism $=(\rho / 2) C_{j}{ }_{j} S_{j}{ }^{X}$

$f_{j}{ }^{Y} \quad$ Vertical drag factor for suspended prism $=(\rho / 2) C_{j}{ }^{Y} S_{j}{ }^{Y}$

$g \quad$ Acceleration due to gravity

$I_{j} \quad$ Component of inertia tensor

$i \quad$ Imaginary unit

$J_{j} \quad$ Component of inertia tensor

$j \quad$ Subscript denoting station number along line

$K_{j} \quad$ Component of inertia tensor

$k_{j+1 / 2}$ Virtual inertia coefficient for segment of cable between stations $j$ and $j+1$

$l_{j+1 / 2} \quad$ Length of line between stations $j$ and $j+1$

$m_{j} \quad$ Mean mass of segments of cable adjoining station $j$

$m_{j}^{*} \quad$ Mass of prism suspended from station $j$

$m_{j}{ }^{\boldsymbol{x}} \quad$ Effective horizontal mass of suspended prism

$m_{j}{ }^{r} \quad$ Effective vertical mass of suspended prism

$q \quad$ Normal conponent of velocity of cable (relative to medium)

$S_{j}{ }^{x} \quad$ Projected area of suspended prism along $x$-axis

$S_{j}{ }^{r} \quad$ Projected area of suspended prism along $y$-axis

$s \quad$ Subscript denoting surface end of line

$T$ Tension

$t \quad$ Time

$u \quad$ Magnitude of velocity of cable (relative to medium)

$V_{j}^{*} \quad$ Volume of prism suspended from station $j$

$V_{j}{ }^{x} \quad$ Equivalent volume of horizontal virtual mass of suspended prism

$V_{j}{ }^{Y} \quad$ Equivalent volume of vertical virtual mass of suspended prism

$W_{j} \quad$ Mean net weight of segments of cable adjoining station $j$

$W_{j}^{*} \quad$ Net weight of prism suspended from station $j$

$X \quad$ Horizontal component of resultant external force

$X_{j}{ }^{*} \quad$ Horizontal component of damping force on suspended prism

$x \quad$ Horizontal coordinate of cable

$Y \quad$ Vertical component of resultant external force

$Y_{j}^{*} \quad$ Vertical component of damping force on suspended prism

$y \quad$ Vertical coordinate of cable

$\mu_{j+1 / 2} \quad$ Linear density of segment of cable between stations $j$ and $j+1$

$\rho \quad$ Density of fluid medium

$\sigma_{j+1 / 2}$ Cross-section area of segment of cable between stations $j$ and $j+1$ Dot signifies differentiation with respect to time.

3. Sample Calculation. A typical calculation was carried out to determine the forces acting on a mooring line, anchored at one end and fastened at the other end to a ship which is in motion in conformity with the wave pattern of the sea. The mooring line was composed of two sections with different elastic and inertial properties and with a heavy weight attached at a position ninety feet from the anchor. 


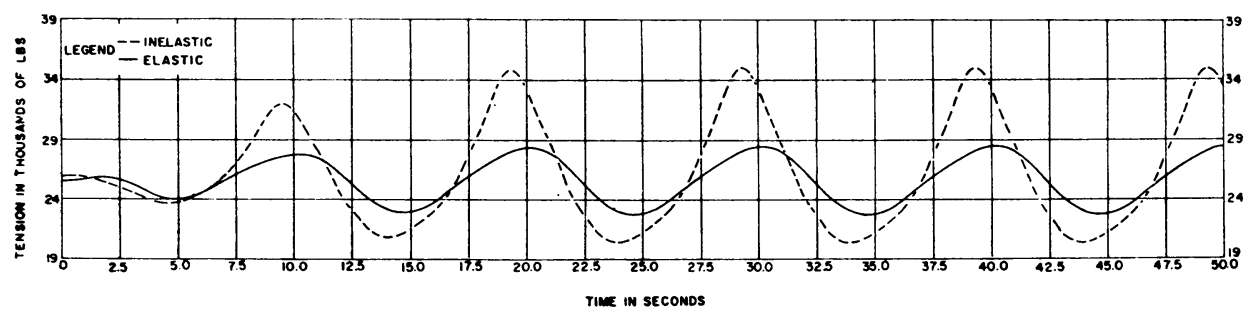

FIG. 1.-Maximum tension in elastic and inelastic motion of a submerged cable.

The motion of the ship was assumed to have an amplitude of six feet and a period of ten seconds. Figure 1 shows the maximum tension in the cable (which occurred at the anchor) plotted as a function of time. Also plotted on the same graph is the maximum tension attained as a result of calculation based on the assumption that the cable is inextensible. It will be noted that the tension is considerably lower when the elastic properties of the cable are taken into consideration.

The calculations were carried out by the method of finite differences in which the time derivatives were replaced by equivalent difference expressions. An appropriate integration interval was chosen to insure stability of the calculation. Prior to the start of calculations the equilibrium conditions of the immersed cable were computed and used as initial conditions in the calculation of the transient motion. The free end of the cable was assumed to start from rest and gradually approach a trochoidal wave motion in accordance with the following set of equations:

$$
\begin{aligned}
& x_{s}(t)=x_{s}^{0}+\left[1-\left(1+\nu_{s} t\right) e^{-\nu_{s} t}\right]\left[a_{s} \cos \omega_{s} t+b_{s} \sin \omega_{s} t\right] \\
& y_{s}(t)=y_{s}{ }^{0}+\left[1-\left(1+\nu_{s} t\right) e^{-\nu_{s} t}\right]\left[c_{s} \cos \omega_{s} t+d_{s} \sin \omega_{s} t\right]
\end{aligned}
$$

where $\nu_{s}, \omega_{s}, a_{s}, b_{s}, c_{s}, d_{s}$ are chosen parameters. An IBM 704 calculator was used to carry out the computation. The computer time required was considerable, primarily as a result of the very small time interval which was necessary to insure stability.

In addition to the plot of maximum tension shown in Figure 1, plots of the tension as a function of position along the cable were obtained at desired time increments. These have been obtained on a film which can be used to produce a "moving picture" of the tension as it varies along the cable as a function of time.

Applied Mathematics Laboratory

David Taylor Model Basin

Washington 7, D. C.

1. Thomas S. Walton \& Harry Polachex, "Calculation of transient motion of submerged cables," $M T A C$, v. 14, 1960, p. 27-46. 